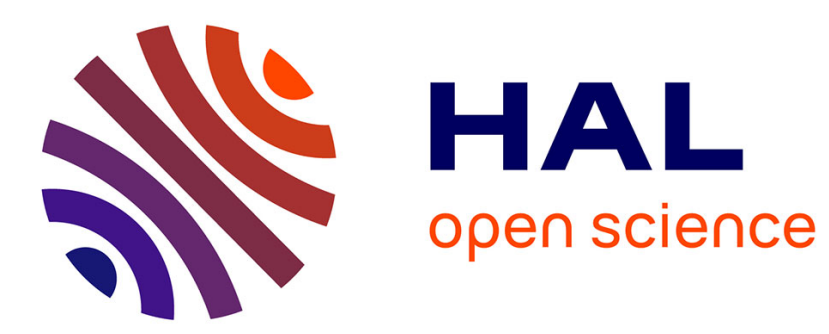

\title{
Influence of orientation and number of layers on the elastic response and failure modes on CLT floors: modeling and parameter studies
}

\author{
Lorenzo Franzoni, Arthur Lebée, Florent Lyon, Gilles Forêt
}

\section{To cite this version:}

Lorenzo Franzoni, Arthur Lebée, Florent Lyon, Gilles Forêt. Influence of orientation and number of layers on the elastic response and failure modes on CLT floors: modeling and parameter studies. European Journal of Wood and Wood Products, 2016, 10.1007/s00107-016-1038-x . hal-01306593

\section{HAL Id: hal-01306593 \\ https://hal.science/hal-01306593}

Submitted on 25 Apr 2016

HAL is a multi-disciplinary open access archive for the deposit and dissemination of scientific research documents, whether they are published or not. The documents may come from teaching and research institutions in France or abroad, or from public or private research centers.
L'archive ouverte pluridisciplinaire HAL, est destinée au dépôt et à la diffusion de documents scientifiques de niveau recherche, publiés ou non, émanant des établissements d'enseignement et de recherche français ou étrangers, des laboratoires publics ou privés. 


\title{
Influence of orientation and number of layers on the elastic response and failure modes on CLT floors: modeling and parameter studies
}

\author{
Lorenzo Franzoni · Arthur Lebée · Florent Lyon · Gilles Foret
}

Received: date / Accepted: date

\begin{abstract}
In the present paper, the bending behavior of Cross Laminated Timber panels is investigated by means of the linear elastic exact solution from Pagano (1970; 1969). The resulting stresses are the input for a wood failure criterion, which can point out the first-crack load and the respective dominant failure mode. Heterogeneous layers are modeled as equivalent and homogeneous layers. This simplified and deterministic modeling gives results in good agreement with a reference experimental test. A comparison is made with respect to the panel's global stiffness and failure stages within the apparent elastic stage. Finally, parameter studies are carried out, in order to quantify CLT limitations and advantages. The effect of varying properties like the panel's slenderness, orientation of transverse layers and number of layers for a fixed total thickness are investigated.
\end{abstract}

\section{Introduction}

Cross Laminated Timber (CLT) panels are relatively new wooden structural products consisting in lumber layers glued on their lower and upper faces and stacked crosswise. The combination of easy-assembling, and high in- and outof plane stiffnesses makes this product competitive. Moreover, the crosswise orientation of wooden layers ensures uniform shrinkage and swelling properties. These qualities allow their use for prefabricated floors, walls and even whole structures, in a new, innovative and low-environmental im-

L. Franzoni · A. Lebée $(\bowtie) \cdot$ G. Foret

Université Paris-Est, Laboratoire Navier (UMR 8205), CNRS, ENPC, IFSTTAR, F-77455 Marne-la-Vallée, France

E-mail: arthur.lebee@enpc.fr

F. Lyon

Centre Scientifique et Technique du Batiment (CSTB), F-77455 Marne-la-Vallée, France pact way of building. In the last 20 years CLT applications have increased in use from small residential houses to more than 8-story buildings. Although recently several standards have been developed or updated for this product (ANSI/APA 2012; DIN 2004) and some design manuals have been published (Schickhofer et al 2009; Gagnon and Pirvu 2013), there is still a need for efficient tools to assess structural efficiency in complex situations. CLT panels can be used as roofs or slabs and therefore submitted to out-of-plane loads. The main structural issue related to their bending behavior is the low transverse shear strength of cross layers. The resulting effect is a rotation of the wood's fibers which leads to the so-called rolling shear brittle failure. This phenomenon becomes more relevant when decreasing the slenderness ratio $L / h$ ( $h$ is the plate's thickness and $L$ the span). During last years a significant number of research studies have been carried out on rolling shear effects in wood and in CLT (Zhou et al 2014; Mestek 2011; Blass and Fellmoser 2004b; Aicher and Dill-Langer 2000). This demand in research clearly calls for a method to analyze CLT panels in bending, which can well predict the transverse shear effects.

CLT plates in bending are affected by heterogeneities at different levels. First, at the material scale, timber features a pronounced variation in mechanical properties. Then, within each layer, two other kinds of heterogeneities can be differentiated. The local orientation of orthotropic coordinate system is a priori unknown due to the variation of annual ring pattern. Moreover, each layer of CLT is made of wooden boards placed side by side, which can be glued or not on their lateral faces, depending on the fabrication process. The resulting discontinuities in the non-gluing case have a nonnegligible influence on the mechanical response, as the studies in Sebera et al (2013) showed. At the structural level, additional heterogeneities can be added in order to meet acoustic and structural needs. Indeed, CLT panels having voids filled by insulating materials are already in production. In 
this paper, the study is limited to "low layer heterogeneity", characterized by only a small spacing of lateral boards. CLT panels having periodic spaces will be the object of further studies.

At the layer scale, an "equivalent-layer" mechanical model is suggested to take into account the CLT layer's heterogeneities, leading to simplified hypotheses on layer behavior. Concerning the structural heterogeneity given by the multi-layer composition, there are currently three main design approaches for CLT in bending: the k-method (Blass and Fellmoser 2004a), the $\gamma$-method EN (2004) and the shear analogy method proposed by Kreuzinger (1999). These theories give acceptable results and some of them have already been adopted by standard design codes (EN 2004; DIN 2004). However their limits lie in a simplified approach, which does not ( $k$-method) or partially ( $\gamma$-method) take into account transverse shear effects or reduce the $2 \mathrm{D}$ problem into a 1D one. Furthermore, all these solutions are suitable only for orthotropic $\left(0^{\circ}\right.$ or $\left.90^{\circ}\right)$ lay-up. Starting from these inadequacies in existing methods, Sturzenbecher et al (2010) showed that high-order plate theories can be used to accurately predict the mechanical behavior of CLT panels in bending. A good match between the predicted deflections and the results of an experimental test available in the literature was found. In the present paper, the exact 3D theory for laminated plates in bending (Pagano 1970; 1969) is applied to CLT panels, in order to predict their mechanical response and to analyze plates with non-orthotropic layups. Being a 3D solution, Pagano's theory can give a better estimation of stress distribution across the panel's thickness than simplified approaches, especially when the panel is submitted to concentrated loads. The elastic bending solution is therefore combined with a failure criterion for wood, in order to extend the comparison with a reference test in terms of failure stages. In the present paper, the main topic of interest is CLT mechanical behavior at the layer and structure scale, leading to a simplified modeling at the material scale. Whereas the wood variability has not been taken into account in this study, having a detailed description of 3D stress enables the determination of interesting features of CLT mechanical behavior. Indeed, the influence on panel's bending behavior of having edge-glued or unglued layers is pointed out, as well as the effects of varying layers number and orientation.

All the modeling tools are presented in Section 3 after the introduction, in Section 2, of the reference experimental behavior of CLT panels. In Section 4, the comparison between the predicted and actual behavior is made in terms of the plate's global stiffness and variations in failure stages. The good agreement with the reference results has led to an investigation on CLT properties by means of the parameter studies in Section 5. In this final Section, transverse shear effects occurring in CLT in bending are quantified, as well as further CLT advantages deriving from the variation of layer orientation and number.

\section{Reference experimental test}

Hochreiner et al (2013) tested square 3-ply and 5-ply CLT panels with slenderness ratios of 10 and 20 and made of Norway spruce lumber boards of strength class C24 (EN 2009). The plates had their four sides supported and were submitted to concentrated loads at their center. Thanks to a combined measuring system of acoustic emission, LVDTs and accurate cutting of specimens after the failure, progressive failure stages were determined as a function of load levels (Fig. 1). At each failure stage, the corresponding crack type identified by the panel's cutting was assigned. The most complete documentation found in the reference paper is about the so-called specimen "EL4", a three-layer panel of slenderness ratio of 20 . Figure 1 reproduces the reference test result for specimen "EL4" in terms of its load-displacement curve, failure stages and respective failure modes. The plate showed a global ductile behavior after the elastic limit, due to its bi-axial bending configuration, whose effect is to redistribute the stresses after the first cracks appear. This "system" effect is particularly evident in Stage 2, where the global linear behavior is not really affected by the appearance of rolling shear cracks.

All the cracking modes found during the loading process are presented in Figure 2. The cracks appearing first called "RS" and "EG" stand respectively for rolling shear failure in cross-layers and edge-gluing failure between lateral boards

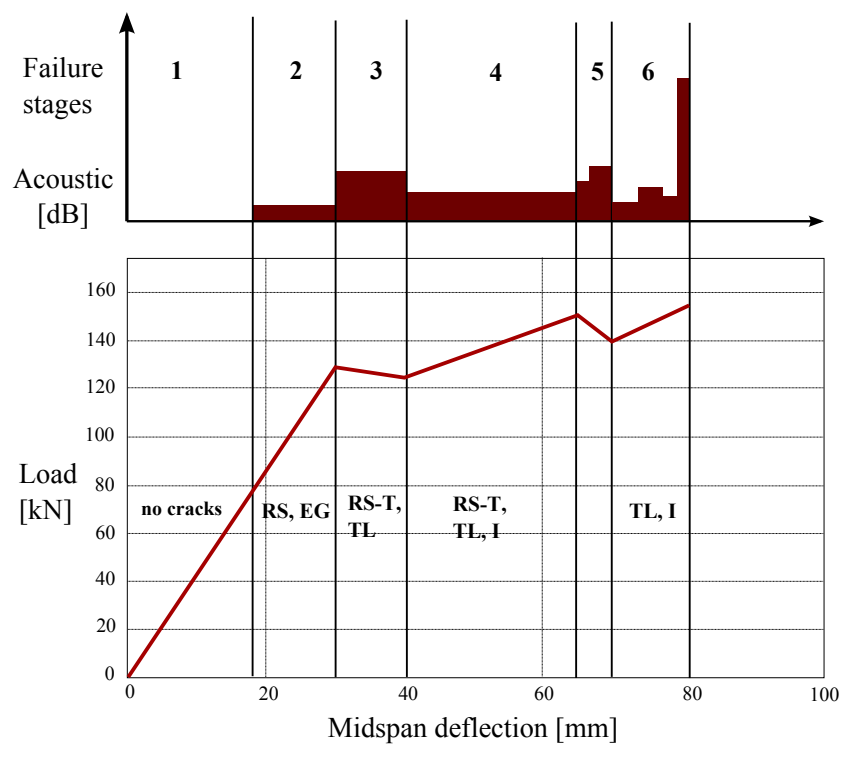

Fig. 1: Schematic load - displacement curve with identified failure stages and associated crack modes found by Hochreiner et al (2013) 


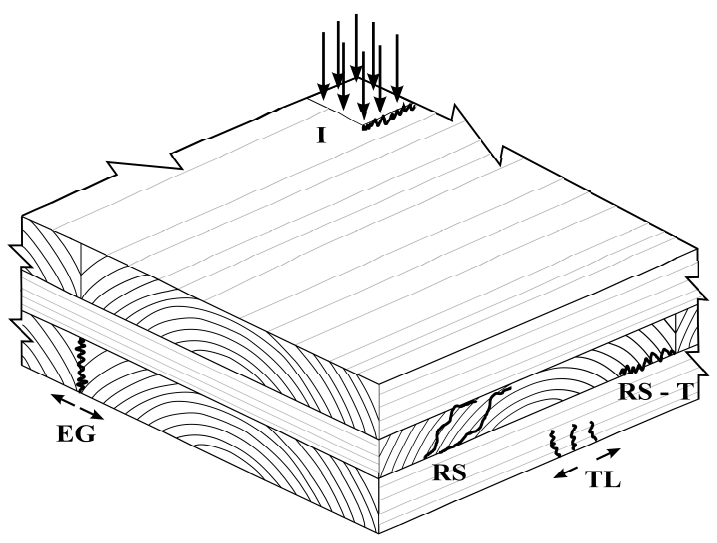

Fig. 2: Failure modes of specimen EL4 (Hochreiner et al 2013)

of the same layer. The failure mode denoted "TL" is the tensile failure in direction parallel to grain, while "I" is the local indentation perpendicular to grain. The failure called "RS $\mathrm{T}$ " is a complex cracking pattern occurring in cross layers at the end of the elastic limit and assumed to derive from interactions between rolling shear and tensile stresses. Moreover, the "RS - T" cracking pattern was also a consequence of the geometrical discontinuities appearing between boards of the same layer, after edge-gluing failure.

The comparison between the predicted and experimental bending response is made in terms of the panel's global stiffness in the linear elastic load stage (stage 1 in Fig. 1) and the variation of failure modes within the apparent elastic stage (until stage 3 in Fig. 1).

\section{Modeling of CLT panels bending behavior}

At the material scale, wood is considered as an elastic, brittle and homogeneous material. The heterogeneities characterizing the CLT layers are taken into account by means of an equivalent and homogeneous layer, whose mechanical properties are defined both in terms of elasticity and failure. Once the simplified mechanical behavior is set, the exact 3D solution in elasticity for laminated plates in bending (Pagano $1970 ; 1969)$ is chosen in order to obtain precise estimation of the plate's mechanical response. Finally, a failure criterion suitable for wood (van der Put 1982) can point out the failure load and the corresponding dominant failure mode of CLT panels in bending.

\subsection{Mechanical behavior of solid wood}

Wood is an orthotropic material with three principal axes. The first one is aligned in the fiber or trunk direction (longitudinal direction, $L$ ). In the transverse plane, the remaining two axes are orthogonal to growth rings (radial direction, $R$ ) and tangential (tangential direction, $T$ ) to them. Figure 3 presents the axes of orthotropy of solid wood. The chosen wood species is Norway spruce (Picea abies), since it is the most widely used wood species in Europe for CLT production and was also used in the reference experiment. The mechanical properties are chosen on the basis of tests in literature on specimens of clear spruce, without knots. Table 1 shows the elastic and strength properties of Norway spruce taken respectively from Keunecke et al (2008) and Dahl (2009). The first subscripts $L, T$ and $R$ represent the wood coordinate system, while the following $t$ and $c$ represent tensile or compressive strength. As it will be shown in the next paragraph, the failure analysis requires the complete set of nine spruce strength parameters, whose values are present only in Dahl (2009). Another experimental campaign on clear spruce strengths in the LT plane (Eberhardsteiner 2002) confirms the congruity of the chosen values, especially for the tensile strength. The failure behavior presented in Tab. 1 describes wood's strength with respect only to pure uni-axial stresses. Therefore, in order to perform an exhaustive failure analysis, a mixed failure criterion for wood is required.

\subsection{Van der Put's mixed failure criterion for wood}

Failure criteria define the material failure by means of normalized expressions, which represent the material's strength surface. A stress state, that reaches or exceeds the failure surface, leads to inelastic phenomena such as damage or plas-

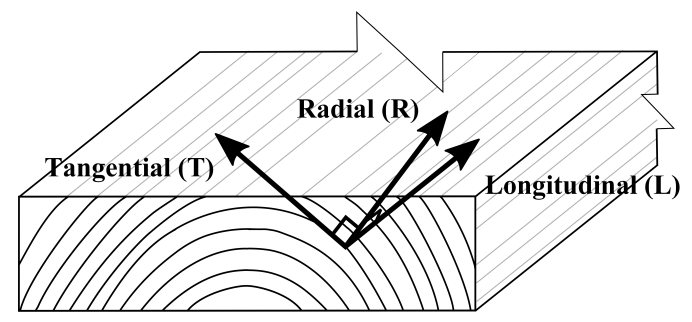

Fig. 3: Material axes of orthotropy for solid wood

Table 1: Elastic and strength properties of Norway spruce [MPa]

\begin{tabular}{cccccccccc}
\hline Elasticity & $E_{L}$ & $E_{T}$ & $E_{R}$ & $G_{R L}$ & $G_{L T}$ & $G_{T R}$ & $v_{L R}$ & $v_{L T}$ & $v_{R T}$ \\
(Keunecke et al 2008) & 12800 & 397 & 625 & 617 & 587 & 53 & 0.36 & 0.45 & 0.48 \\
\hline Failure & $f_{L, t}$ & $f_{L, c}$ & $f_{T, t}$ & $f_{T, c}$ & $f_{R, t}$ & $f_{R, c}$ & $f_{R L}$ & $f_{L T}$ & $f_{T R}$ \\
(Dahl 2009) & 63.4 & 28.9 & 2.8 & 3.8 & 4.9 & 3.6 & 7.1 & 4.8 & 2.0 \\
\hline
\end{tabular}


tic strains. The most widely used isotropic failure criteria are based on von Mises maximum distortion energy. These criteria generally follow a quadratic expression which represents an elliptic surface. Dealing with anisotropic materials, the rotated and translated ellipsoid of Tsai-Wu (Tsai and Wu 1971) is the most common failure surface. The general quadratic expression for orthotropic materials can be written as:

$$
\begin{array}{r}
f(\sigma): A \sigma_{L}^{2}+B \sigma_{T}^{2}+C \sigma_{R}^{2}+D \sigma_{L} \sigma_{T}+E \sigma_{L} \sigma_{R}+ \\
+F \sigma_{T} \sigma_{R}+G \sigma_{L}+H \sigma_{T}+I \sigma_{R}+ \\
+L \tau_{L T}^{2}+M \tau_{L R}^{2}+N \tau_{R T}^{2}=1.0
\end{array}
$$

where $\sigma$ and $\tau$ are respectively the longitudinal and shear stresses in orthotropic coordinates $L, T$, and $R$. The capital letters are function of the material strengths and they determine the geometry of the failure surface. While the coefficients of quadratic terms $(A, B, C$ and $L, M, N)$ represent the semi-axes of the elliptical surface, linear $(G, H, I)$ and interaction $(D, E, F)$ terms in (3.1) respectively translate and rotate the ellipsoid. The value of interaction terms in failure criteria for anisotropic materials is still nowadays under discussion. During his studies on failure criteria for wood, van der Put (1982) showed how the value of the interaction term has a negligible influence when the stress path is closed to the failure surface. Therefore, Van der Put's failure criterion is a function $f(\sigma)$ like (3.1) but without interaction terms. This failure criterion has been compared in Cabrero et al (2012) with other criteria applied to spruce failure, and it turned out to be one of the most predictive. Therefore, considering also its simple implementation due to the absence of interaction terms, Van der Put's failure function has been chosen for the failure analysis. The actual values of the strength coefficients of (3.1) as function of the strength moduli are presented in Appendix A of the Online Resource.

If the failure criterion is proportional to the applied load, it is straightforward to find the failure load from a single linear solution. Whereas $\sigma$ is already proportional to the applied load, the function $f(\sigma)$ derived by van der Put (1982) turns out not to be proportional. Hence, it is necessary to derive a new function $F(\sigma)$, which will describe the same failure surface, but also satisfy the condition

$F(\lambda \sigma)=\lambda F(\sigma), \quad \lambda>0$

Property (3.2) ensures the same variation $\lambda$ of $F(\sigma)$, when varying the external load (and the related stress state $\sigma$ ) of a positive quantity $\lambda$. Detailed derivation of the new function $F(\sigma)$ is reported in Appendix A of the Online Resource. The value $\lambda$ represents the multiplier coefficient to reach the failure point. The spatial distribution within the CLT panel of $\lambda(\sigma)$ multiplied by the failure load $F_{c}$ represents the load levels necessary to reach progressive failures under linear elastic hypotheses.

When the failure load is determined, it is of particular interest to establish the associated dominant failure mode. This can be achieved computing the ratios between each of the six stress components expressed in the wood's coordinates $\left(\sigma_{L}, \sigma_{T}, \sigma_{R}, \tau_{L T}, \tau_{R L}, \tau_{T R}\right)$ and their respective strengths $\left(f_{L, c-t}, f_{T, c-t}, f_{R, c-t}, f_{L T}, f_{R L}, f_{T R}\right)$. The maximum value of these ratios can point out the dominant failure mode. This ratio is computed at any point within the panel.

\subsection{Equivalent CLT Layer model}

Both complete (3D) and reduced (2D or 1D) solutions for layered plates in bending consider every layer as homogeneous. In practice, each CLT layer is made of boards placed side by side and it is affected by the heterogeneities presented before. Hence, it is necessary to set a homogeneous "Equivalent CLT Layer" model, which could take such heterogeneities into account. As presented before, the first complexity derives from the variation of growth rings' orientation inside each layer, which leads to an unknown orientation of the local orthotropic coordinate system.

Moreover, in case of unglued lateral boards, the resulting discontinuities influence the equivalent layer. Note that, if the lateral edges of boards were initially glued, the experimental evidence showed that the edge gluing detachment is one of the first failure modes (see Section 2).

\subsubsection{Continuous Equivalent Layer}

If the boards' lateral edges are glued, each wooden layer can be viewed as a continuous layer. The same material behavior in directions $N$ and $Z$ of the board's reference frame (Fig. 4) is considered, in order to overcome the irregularity of growth rings. While the $Z$ direction remains always the same, directions $L$ and $N$ change together with the orientation of the considered layer. Table 2 presents the elastic and strength properties of the continuous Equivalent Layer. The defined elastic moduli for the $N$ or $Z$ direction are the mean values between the corresponding $T$ and $R$ ones for solid

Table 2: Elastic and strength properties of a continuous CLT layer [MPa]

\begin{tabular}{cccccccccc}
\hline Elasticity & $E_{L}$ & $E_{N}$ & $E_{Z}$ & $G_{Z L}$ & $G_{L N}$ & $G_{N Z}$ & $v_{L Z}$ & $v_{L N}$ & $v_{Z N}$ \\
& 12800 & 511 & 511 & 602 & 602 & 53 & 0.41 & 0.41 & 0.48 \\
\hline \multirow{2}{*}{ Failure } & $f_{L, t}$ & $f_{L, c}$ & $f_{N, t}$ & $f_{N, c}$ & $f_{Z, t}$ & $f_{Z, c}$ & $f_{Z L}$ & $f_{L N}$ & $f_{N Z}$ \\
& 63.4 & 28.9 & 2.8 & 3.6 & 2.8 & 3.6 & 4.8 & 4.8 & 2.0 \\
\hline
\end{tabular}


Table 3: Elastic and strength properties of a discontinuous CLT layer [MPa]

\begin{tabular}{cccccccccc}
\hline Elasticity & $E_{L}$ & $E_{N}$ & $E_{Z}$ & $G_{Z L}$ & $G_{L N}$ & $G_{N Z}$ & $v_{L Z}$ & $v_{L N}$ & $v_{Z N}$ \\
& 12800 & 0.0 & 511 & 602 & 602 & 53 & 0.41 & 0.0 & 0.0 \\
\hline \multirow{2}{*}{ Failure } & $f_{L, t}$ & $f_{L, c}$ & $f_{N, t}$ & $f_{N, c}$ & $f_{Z, t}$ & $f_{Z, c}$ & $f_{Z L}$ & $f_{L N}$ & $f_{N Z}$ \\
& 63.4 & 28.9 & - & - & 2.8 & 3.6 & 4.8 & 4.8 & 2.0 \\
\hline
\end{tabular}

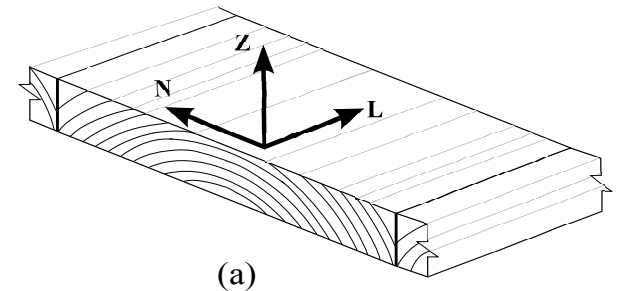

(a)

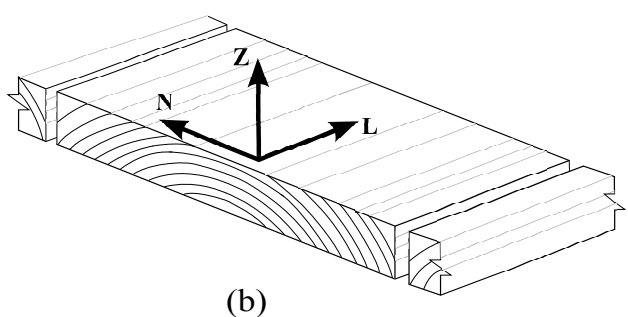

Fig. 4: Schematic continuous (a) and discontinuous (b) Equivalent CLT Layers together with the layer's reference frame

wood (Tab. 1), while the strength parameters are the lower values.

\subsubsection{Discontinuous Equivalent Layer}

When CLT boards are not glued together on their lateral edges, or when in-plane stress caused the edge-gluing detachment, each layer becomes discontinuous (Fig. 4b) and such discontinuities preclude any transmission of stresses between separated boards. This also means that failure in $\mathrm{N}$-direction cannot occur. Table 3 shows the considered mechanical behavior of an equivalent and discontinuous layer made of Norway spruce. Intuitively, due to the gaps between lateral boards, the equivalent layer's plane shear modulus $G_{L N}$ may be set to zero (Mestek et al 2008). However, all layers are glued on their upper and lower faces and hence the discontinuous CLT panel has a (reduced) in-plane shear stiffness (Moosbrugger et al 2006). The same conclusion can be deduced for the plane shear strength of a discontinuous layer. A more accurate investigation on the actual plane shear behavior of a discontinuous layer will be the object of further studies. In this paper, it is assumed that the in-plane shear behavior of layers equals the wood's behavior. The Poisson's ratios $v_{L N}$ and $v_{Z N}$ represent the layer's strain in direction $N$, due to the imposed strain in directions $L$ and $Z$, respectively. Considering layers with discontinu- ities along direction $N$ as in Figure $4 \mathrm{~b}$, the values of these coefficients are assumed to be zero.

\subsection{Pagano's exact solution for laminates in bending}

Once the model for an equivalent and homogeneous CLT layer is set, the analytical bending solution can be chosen between complete or reduced approaches. 1D theories (Blass and Fellmoser 2004a; EN 2004; Kreuzinger 1999) have very low computational costs but give approximate results. 2D plate theories for laminates in bending (Lebée and Sab 2011; Thai et al 2013) are still reduced approaches, but return more precise results than beam theories. Nevertheless, the specimens of the reference test were submitted to concentrated loads, which produce complex stress states close to loading area, difficult to predict with reduced approaches. Therefore, the complete 3D solution from Pagano $(1970 ; 1969)$ was chosen in order to obtain a precise estimation of CLT bending behavior. Pagano derived such solution for plates having homogeneous layers and perfect connections between them under uni-axial (Pagano 1970) or biaxial (Pagano 1969) bending configurations. The bi-axial bending solution is used for the comparison with the reference test, while the uni-axial solution is applied to the parameter studies on CLT properties.

\subsubsection{Uni-axial bending}

The most common bending configuration for structural panels is represented by a plate simply supported on two sides. Pagano's 3D solution for layered plates in uni-axial bending represents such a bending configuration. A plate under uni-axial, or cylindrical, bending has only two sides supported along the same direction, while the other direction is assumed as infinite and there are no boundaries (Fig. 5a). The displacement field is assumed to be a single Fourier-like series, like the out-of-plane load $p_{3}$, acting on the plate's upper or lower surface. In Pagano's uni-axial bending, the only imposed condition on the bounded edges is zero vertical displacement $u_{3}$, leaving free the in-plane displacements.

\subsubsection{Bi-axial bending}

CLT panels tested in the framework of the reference experiment were supported on their four sides, which corresponds to the bi-axial bending solution from Pagano. This solution 


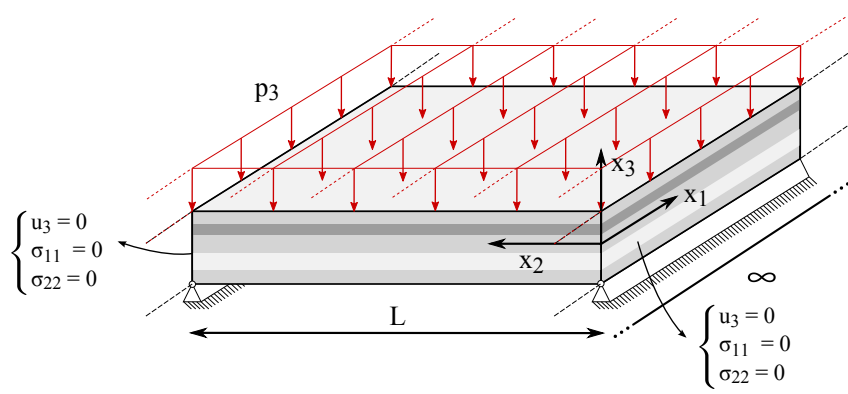

(a)

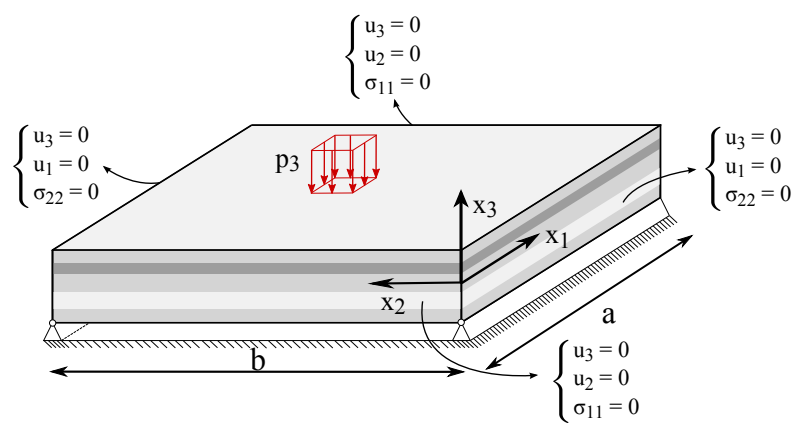

(b)

Fig. 5: Pagano's uni-axial (a) and bi-axial (b) bending configurations with corresponding applied loads and boundary conditions. $u_{1}, u_{2}$ and $u_{3}$ stand for the displacements in directions $x_{1}, x_{2}$ and $x_{3}$ respectively

is valid for rectangular orthotropic plates, whose axes are aligned with the axes of the supports. In this case, all the plate's sides are simply supported (Fig. 5b) and the displacements as well as the surface load $p_{3}$ are expressed as double Fourier-like series. The boundary conditions, which make possible Pagano's solution in bi-axial bending, consist in restraining vertical and tangential displacements at the plate's bounded sides. As Fig. 5b shows, the tangential displacements for edges along direction 2 and 1 are respectively $u_{2}$ and $u_{1}$. However, since the tangential displacements of the reference panel's edges were not restrained, the experiment configuration has more degrees of freedom than the bending solution. Further analyses not reported in this paper showed that the reference slenderness ratio of 20 gives a difference of about $10 \%$ between the estimated deflection preventing or not edges' tangential displacements. Hence, when choosing Pagano's bi-axial solution to reproduce the reference test, it is expected to find a global stiffness about $10 \%$ higher than the reference one.

\section{Comparison with the reference test}

In this Section, the predicted bending behavior is compared with the reference behavior in terms of the panel's global stiffness and failure stages.

\subsection{Global stiffness}

Table 4 shows the plate's global stiffness comparison between the reference test and each model for an equivalent CLT layer. When the lateral boards are glued to each other, the panel is continuous and its global stiffness is the slope of the load-displacement curve in the proportional limit (from 0 to $50 \mathrm{kN})$. Then, when the edge-gluing detachment occurs $(\approx 80 \mathrm{kN})$, the panel presents gaps between boards and its stiffness slightly decreases. For each case, the corresponding equivalent-layer model is used to predict the CLT plate's global stiffness. Because of the experimental uncertainty, a $5.0 \%$ margin of error in measuring the reference value of the plate's stiffness is assumed. Taking into account the discrepancy between modeled and actual boundary conditions described in 3.4.2, a predicted plate's stiffness about $10 \%$ greater than the reference stiffness is expected. Indeed, as Table 4 shows, for both the continuous and discontinuous case, the predicted global stiffnesses are about $10 \%$ higher than the reference stiffness. A CLT plate made of continuous layers shows higher stiffness compared to the one predicted using a discontinuous model, due to the absence in the latter of any contribution of fibers along direction $N$ (see Tab. 3). However, the hypotheses made on elastic properties of continuous and discontinuous CLT layers lead to a relatively small difference between their elastic response in terms of vertical displacements.

Table 4: Plate's global stiffness comparison

\begin{tabular}{lcc}
\hline Continuous panel $(0-50 \mathrm{kN})$ & Global Stiffness $\left[\frac{\mathrm{kN}}{\mathrm{mm}}\right]$ & measuring error \\
\hline Experimental reference & 4.60 & $\pm 5.0 \%$ \\
Predicted & $5.03(+9.3 \%)$ & - \\
\hline Discontinuous panel $(80-120 \mathrm{kN})$ & Global Stiffness $\left[\frac{\mathrm{kN}}{\mathrm{mm}}\right]$ & measuring error \\
\hline Experimental reference & 4.14 & $\pm 5.0 \%$ \\
Predicted & $4.60(+11.1 \%)$ & - \\
\hline
\end{tabular}




\subsection{Failure stages comparison}

In addition to the elastic panel's deflection, Pagano's solution can precisely estimate the stress distributions within the panel. In Section 3.2, the identification of failure load and the corresponding dominant failure mode are described. A progressive increase in the load after the first failure, leads to a proportional variation of the function $F(\sigma)$ within the panel. Therefore, considering simultaneously the spatial distributions of the failure criterion $F(\sigma)$ and the related failure mode, leads to the derivation of failure stages under linear elastic hypothesis. A preliminary analysis revealed that, when considering the plate's cross-section, the first failures take place under the concentrated load at the plate's center. Hence, an investigation on the variation of failure modes along the plate's axes of symmetry is sufficient. The reference specimen is a CLT 3-ply and its failure stages were introduced in Section 2. For both continuous and discontinuous cases, the distributions of failure load are plotted for the plate's cross-sections at $x_{1}=a / 2$ and $x_{2}=b / 2$ (see Fig. 5b). This distribution highlights the load level necessary to reach the failure along the plate cross-sections. Over such a distribution, the superimposition of the dominant failure modes points out the progressive failure stages. Table 5 presents the chosen abbreviations for the failure modes within a layer of a CLT panel.
Table 5: Abbreviations of failure modes

\begin{tabular}{lc}
\hline Failure mode & Abbreviation \\
\hline Tensile Longitudinal & L-t \\
Compressive Longitudinal & L-c \\
Tensile Direction-N & N-t \\
Compressive Direction-N & N-c \\
Tensile Direction-Z & Z-t \\
Compressive Direction-Z & Z-c \\
Transverse shear ZN (Rolling Shear) & RS \\
Transverse shear ZL & ZL \\
Plane shear LN & LN \\
\hline
\end{tabular}

\subsubsection{Continuous layer}

Figure 6a shows the distribution of failure load and failure modes within the panel's cross section predicted with a continuous equivalent layer. For a better presentation, the ratio between the panel's thickness and span is scaled at about 10:1. The first failure mode occurs at about $50 \mathrm{kN}$ of load level. Such a failure mode is a perpendicular compressive failure close to the punching area $(\mathrm{N}-\mathrm{c})$, which is actually difficult to observe experimentally. Detailed analyses of stresses revealed how this area is affected by a tri-axial stress state. Therefore, the contribution to material failure derives from all the compression components in direction $L, N$ and $Z$, with the latter as the dominant one. This is a very local phenomenon not affecting the linear response of the panel at a very short distance from the punch. Moreover, the punch, being modeled as a uniformly distributed load, cannot take into account the actual contact phenomena oc-

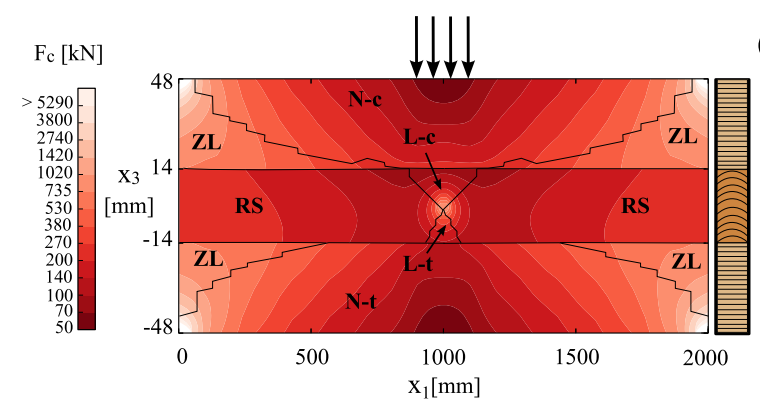

(a)
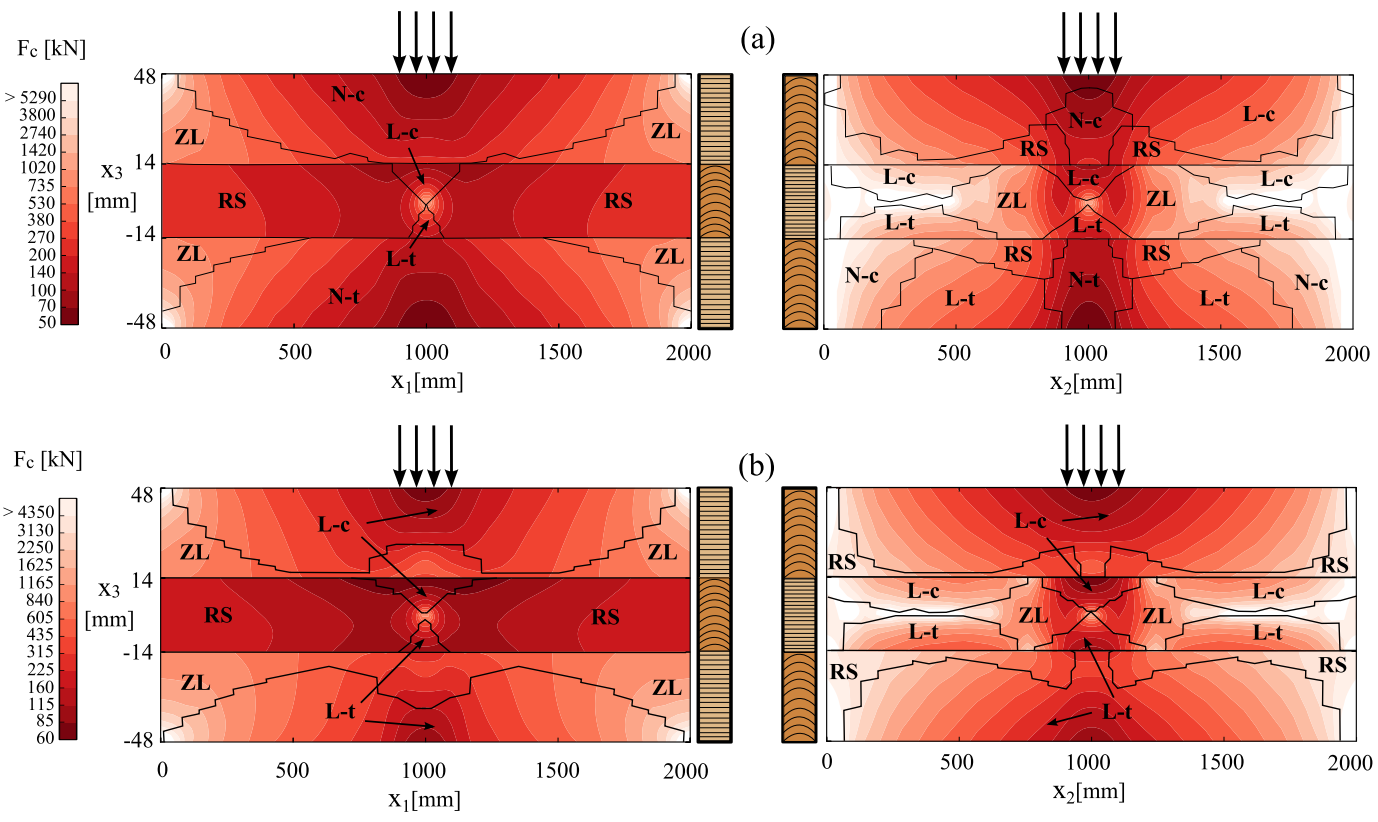

(b)

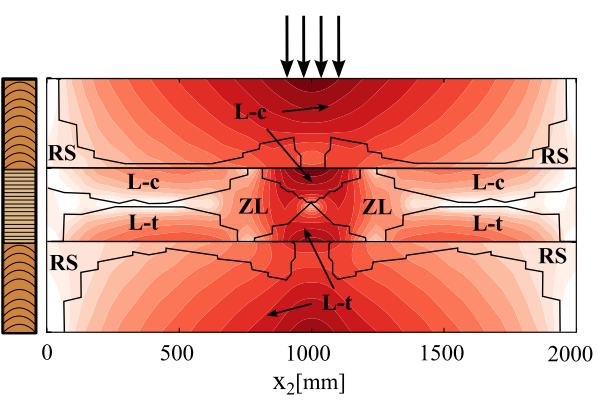

Fig. 6: Variation of failure load and failure modes at $x_{1}=a / 2$ and $x_{2}=b / 2$ inside the panel predicted with the continuous (a) and discontinuous (b) models 


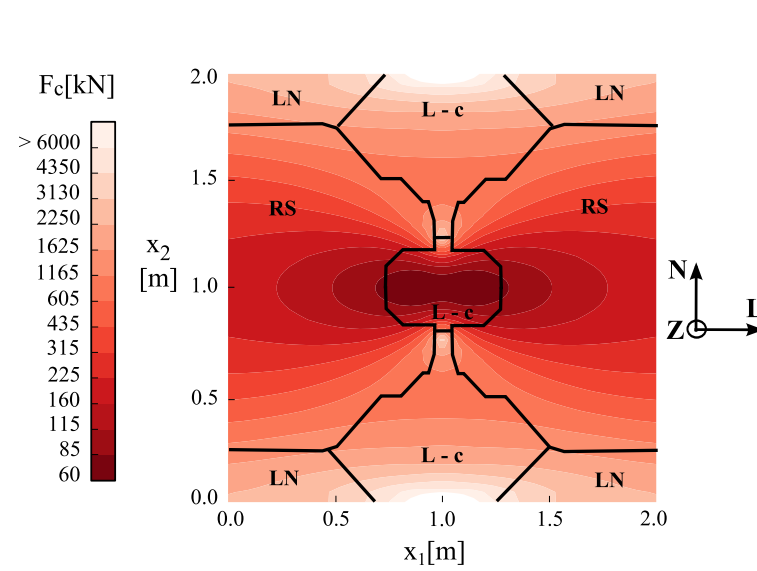

(a)

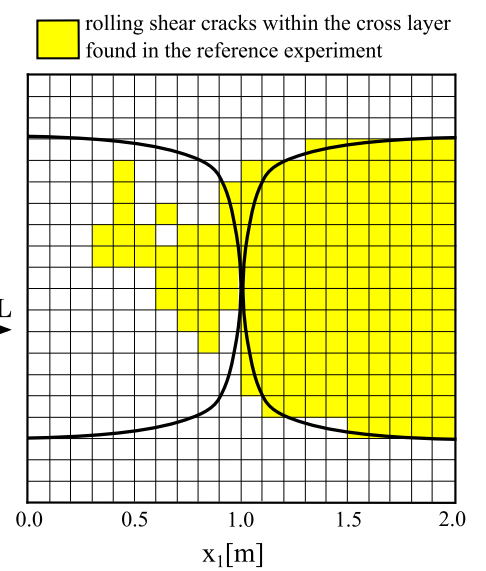

(b)

Fig. 7: Horizontal section of the plate at $x_{3}=+13 \mathrm{~mm}$. Failure stages predicted with the discontinuous model (a) and the corresponding cracking pattern found in the reference experiment (b)

curring in the experiment. The subsequent failure stage is tensile failure of the bottom layer in direction $N(\mathrm{~N}-\mathrm{t} \approx 70$ $\mathrm{kN})$. Such a predicted failure could explain the corresponding edge-gluing (EG) separation found in the experimental test at similar load levels (see Section 2) along direction $N$ of boards. Rolling shear failure of cross layers $(\mathrm{RS} \approx 90 \mathrm{kN})$ and longitudinal tensile failure of bottom layer $(\mathrm{L}-\mathrm{t} \approx 100$ $\mathrm{kN}$ ) are the next failure modes. Both of them are estimated at load levels, which slightly deviate from the experimental evidence, especially the tensile failure.

\subsubsection{Discontinuous layer}

Since the first significant observed damage is the edgegluing detachment, it is worth investigating a discontinuous equivalent layer. Figure $6 \mathrm{~b}$ presents the predicted failure stages for a discontinuous equivalent layer. As introduced before, the modeling of discontinuous layers prevents wood's failure in $N$-direction (Fig. 4b). Therefore, the first compressive failure close to the punching area is a contribution of only compression in direction $L$ and $Z$, where the former is the dominant one (L-c $\approx 60 \mathrm{kN})$. Again, the modeling of wood's mechanical behavior led to a compressive failure close to the punching area, which does not affect the plate's global behavior. Rolling shear failure in the cross layer is the following predicted failure, with a corresponding load level $(\mathrm{RS} \approx 80 \mathrm{kN})$ in accordance with the reference test. Moreover, the propagation of such a failure from the plate's center to its' edges is in agreement with the experimental evidence, as Figure 7 shows. Finally, the predicted failure stage at 120 $\mathrm{kN}$ is longitudinal tensile failure in the $L$-direction (L-t) of the bottom layer, which is in a good agreement with the reference behavior (see Fig. 1).

\subsubsection{Discussion}

Table 6 summarizes the predicted failure stages with continuous or discontinuous CLT layer and compares them to the experimental evidence. As for the elastic stiffness comparison, each equivalent-layer model is in accordance within ranges of load levels, which correspond to glued or unglued lateral boards. Indeed, the continuous CLT layer gives good prediction on failure modes at low load levels, when the narrow boards are still glued. Notwithstanding the compressive indentation under the punch, the first predicted failure in this stage is tensile failure in the tangential direction within the bottom layer, which could cause the actual edge-gluing failure of neighboring layers found in the experiment. The discontinuous model fits well the experimental evidence at higher load levels, where the actual rise of rolling shear and tensile failures are predicted with a more accurate precision than in a continuous geometry. Finally, it appears that the edge-gluing of narrow boards makes the panel a little stiffer but, being the first failure mode, the already "damaged" discontinuous model gives better prediction of global load-carrying capacity of the panel. Therefore the discontinuous equivalent-layer model will be used for the parameter

Table 6: Summary of predicted failure stages in comparison with the experimental evidence

\begin{tabular}{cccc}
\hline Load level $[\mathrm{kN}]$ & \multicolumn{3}{c}{ Failure modes } \\
\hline & Reference test & Continuous & Discontinuous \\
60 & - & N-c & - \\
70 & - & N-c & L-c \\
80 & - & N-t & L-c \\
90 & RS/EG & N-t & RS \\
100 & RS/EG & RS & RS \\
120 & RS/EG & L-t & RS \\
& L-t & L-t & L-t \\
\hline
\end{tabular}


studies on CLT properties presented in the next section.

Like every modeling procedure, the predicted bending behavior with the present model depends on the chosen mechanical properties of raw materials. Surprisingly, elastic and strength parameters of clear spruce lead to an accurate prediction of the experimental bending behavior, even if the reference CLT panel was made of boards of strength class C24 having knots. When dealing with CLT in bending, elastic and strength responses mainly derive from either tensile or (rolling) shear effects (Mestek 2011; Hochreiner et al 2014; Czaderski et al 2007), as the previous Section 4.2 also shows. While rolling shear stiffness and strength are commonly assumed to be independent from the presence of defects (ETA 2013; Blass and Fellmoser 2004b;a; Blass and Gorlacher 2000), tensile properties strongly depend on lumber strength class. Generally, dealing with C24 strength class, a mean elastic modulus value of 11.0 GPa (EN 2009) and a mean tensile strength of about $30 \mathrm{MPa}$ (Stapel and van de Kuilen 2014) are assumed. Further simulations not reported in this paper showed that when using such mean values of wood having knots, the predicted stiffness and failure stages deviate significantly from the experimental reference. In Section 4, a good agreement between the predicted and actual bending behavior is found using an elastic modulus of $12.8 \mathrm{GPa}$ and a tensile strength of $63 \mathrm{MPa}$. This could be explained by a "system effect" when assembling lumber boards in a CLT configuration, which increases the panel stiffness and tensile strength, as also suggested by Jobstl et al (2006).

\section{Investigation on CLT panel properties}

Since the discontinuous equivalent-layer gives a good prediction of crosslam bending behavior, parametric studies with this model are carried out in order to better understand

CLT properties and quantify their advantages and limits. The considered bending configuration is a uni-axial bending and the out-of-plane load is an evenly distributed load. In this Section only the mechanical and deterministic behavior is considered.

\subsection{Influence of transverse shear effects}

The transverse shear weakness of CLT panels is due to the presence of cross layers and their low shear strength and stiffness. Shear effects in bending elements become more significant, while the slenderness ratio decreases. Figure 8 shows the failure load and mid-span deflection for a 3-ply and 5-ply CLT as a function of the plate's slenderness ratio $L / h$. The total plate's thickness $h$ is assumed to be constant at $20 \mathrm{~cm}$ for both 3-ply and 5-ply panels, while only the plate's span $L$ changes. The slope variation of the failure load trend in Fig. 8a points out the change of failure mode as a function of the slenderness ratio. This derives from the linear and quadratic dependency of, respectively, shear and bending failure load from the slenderness ratio. Moreover, for a plate under a uniform uni-axial load, there is no interaction between bending and shear forces. While for low slenderness ratios the dominant failure mode is rolling shear (RS) in cross layers, when the plate is slender, the bending failure (namely L-c of upper layer) becomes the dominant failure mode. The transition slenderness from bending to shear failure turns out to be 15 for a 5-ply and 19 for a 3-ply. The 5-ply panel, having lower transition slenderness and higher shear failure load, shows less weakness to rolling shear stresses than the 3-ply CLT.

The difference between the deflection predicted using Pagano's solution $\left(U_{3, P a g}\right)$ and the deflection predicted using the Kirchhoff-Love plate theory for laminates (March 1936) $\left(U_{3, \text { Kir }}\right)$ which neglects the shear deformation, can
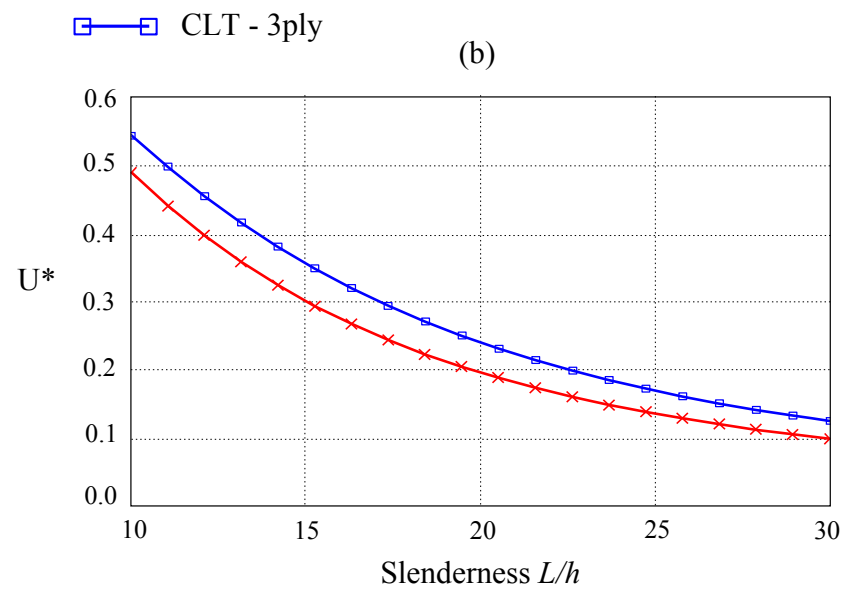

(b)

Fig. 8: Failure load (a) and deflection (b) trends for 3-ply and 5-ply CLT in uni-axial bending when varying slenderness. $U^{*}$ $=\frac{U_{3, P a g}-U_{3, K i r}}{U_{3, P a g}}$

(a)

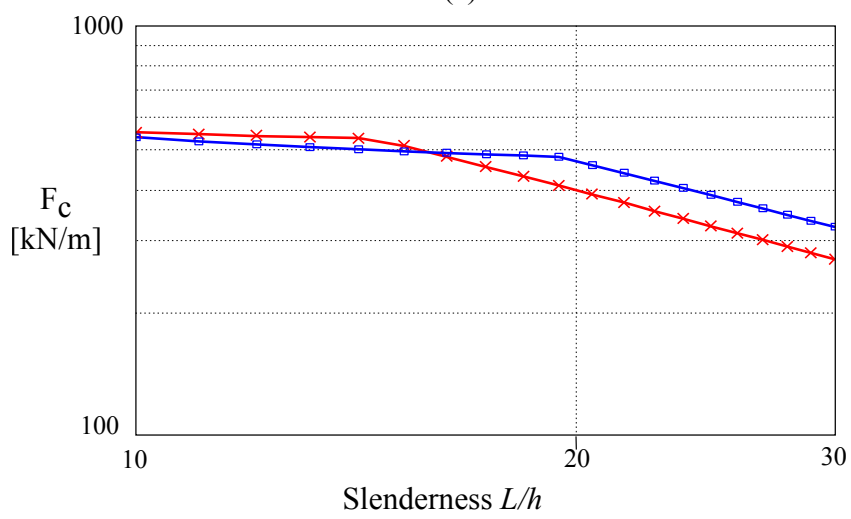


quantify the shear contribution to the total deflection. Figure $8 \mathrm{~b}$ presents the variation of this difference as a function of the CLT panel's slenderness ratio. When the plate's span increases, the increasing slenderness yields a negligible shear deflection and an increasing bending one. However, even when the panel is slender and the failure mode is bending, the shear contribution to deflection is still about 10 $\%$.

5.2 Varying the number of layers for a fixed total thickness

Of particular interest in CLT applications is the optimal number of layers in the cross section to obtain the best mechanical behavior. Therefore, this study aims to show how the CLT mechanical response changes when the number of layers increases from 3 to 23 for a fixed total thickness, which involves a progressive thinning of layers. The results are as follows expressed in terms of the panel's maximum vertical displacement $U_{3}$ and failure load $F_{c}$. The two cases when the plate is thick or slender are presented. For a better visualization, mid-span deflection values are normalized to the Kirchhoff-Love deflection (Kirchhoff 1850) of a corresponding "solid wood" panel $\left(U_{3, s w, \text { Kir }}\right)$ having only one longitudinal layer. Figure 9 presents the variation of failure load $F_{c}$ and mid-span deflection $U_{3}$ as a function of the number of layers for slender and thick CLT plates. The panel's dimensions are $L=5 \mathrm{~m}, h=20 \mathrm{~cm}$ for the panel having a slenderness ratio of 25 and $L=2 \mathrm{~m}, h=20 \mathrm{~cm}$ for the thicker plate. For a plate in uni-axial bending only the layers parallel to the main direction bear the bending stresses, while the transverse layers do not contribute to global stiffness and strength. Hence, the progressive decreasing of the total thickness of longitudinal layers leads to higher deflections and lower failure loads. Unlike the slender case, the variation of failure load when the dominant effect is shear, de- creases with oscillations (see Fig. 9a). This is because CLT panels with a central longitudinal layer (5ply, 9ply etc.) have the cross layers placed at a distance from the cross-section's center (where the shear stresses are maximum), while for the other lay-ups (3ply, 7ply etc.), the central layer is cross and shear-compliant. Therefore, the trend of shear failure load is dependent on the kind of lay-up, in addition to the number of layers within the cross section.

From a deterministic point of view, homogenizing the panel in uni-axial bending by means of progressive thinner layers has a negative impact on the mechanical response. However, taking into account wood's defects such as knots (reliability approach), thinner layers could lead to more uniform CLT mechanical properties.

\subsection{Varying cross layer orientation}

A solution to mitigate CLT shear weakness could be varying the in-plane orientation of transverse layers, in order to change their actual shear stiffness and strength from $T R$ to $R L$. Hence, in this section the effect of a progressive rotation of transverse layers on CLT bending behavior is analyzed. Four different configurations with varying layer lamination have been studied. Configurations called $1 a$ and $1 b$ (Figure 10 - top) are five-layer panels and the difference between them is the opposite orientation of transverse layers. Configuration 2 comes from a study present in literature (Chen and Lam 2013), which involved a 4-ply CLT with the two central layers oriented crosswise at $+45^{\circ}$ and $-45^{\circ}$. Finally, configuration 3 is a 3-ply plate whose central layer has variable orientation. At $\theta=90^{\circ}$, all the configurations have the crosswise CLT lamination, while at $\theta=0^{\circ}$ they behave like a Glued Laminated Timber. Figure 10 illustrates the variation of elastic mid-span deflection $U_{3}$ as a function of the layers' orientation $\theta$ for the four configurations having the same to- (a)

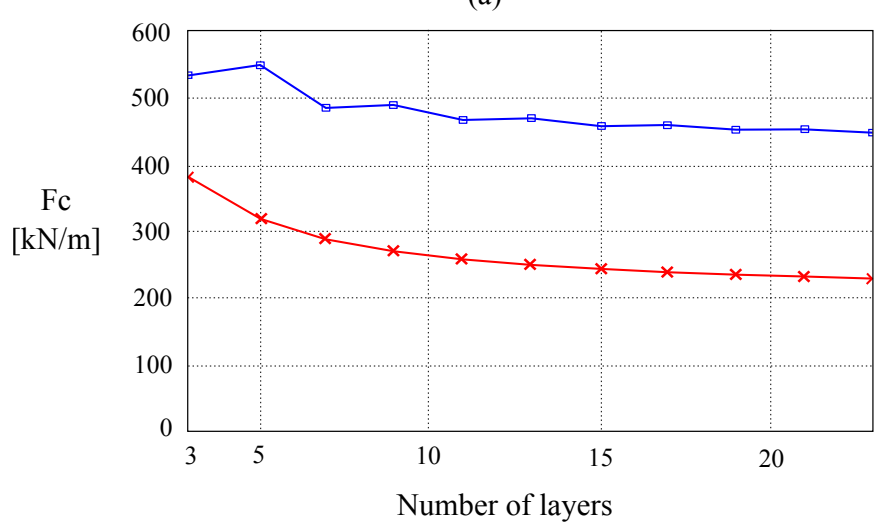

$\square$ Slenderness 10

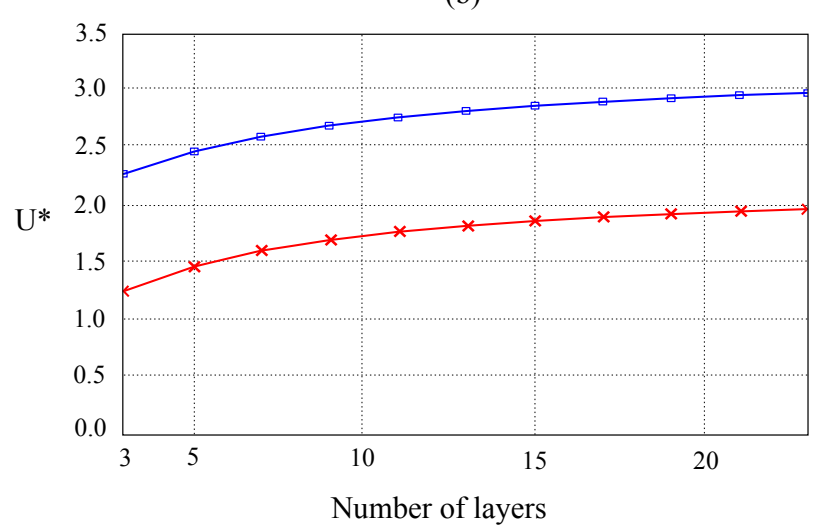

Fig. 9: Variation of CLT failure load $F_{c}$ (a) and normalized mid-span deflection $U_{3}$ (b) as a function of the increasing number of layers for thick and slender panels. $U^{*}=\frac{U_{3, \text { Pag }}}{U_{3, s w, K i r}}$ 

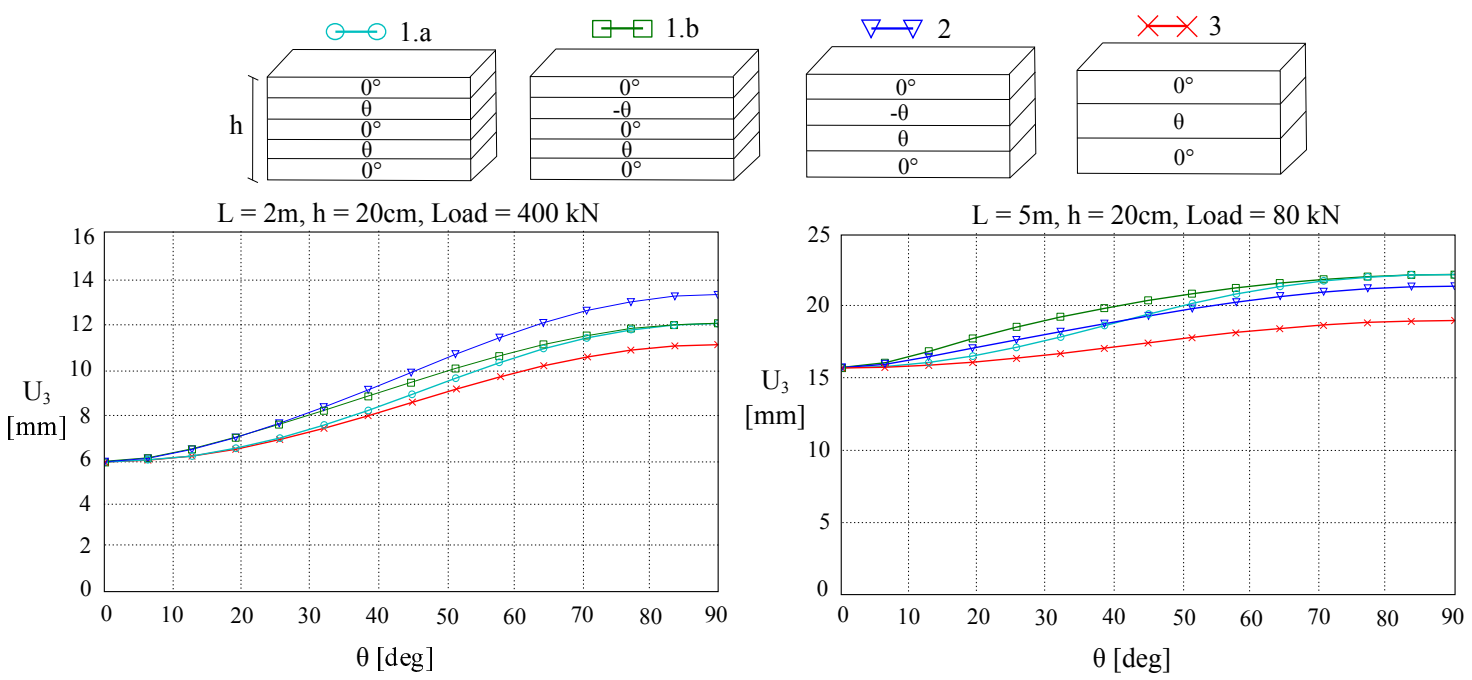

Fig. 10: Variation of deflection $U_{3}$ as a function of layer orientation for slenderness ratios of 10 (left) and 25 (right)

tal thickness $h=200 \mathrm{~mm}$. Both cases of a thick and slender panel are analyzed using respectively a span of $2 \mathrm{~m}$ and $5 \mathrm{~m}$. While changing the panel's lamination from GLT to CLT, the deflection increases. This effect is particularly evident at a low slenderness ratio, showing that a variation of the actual shear modulus from $G_{T R}$ to $G_{R L}$ mitigates the shear deflection. The influence of the cross layers' direction on the panel's deflection is lower when increasing the slenderness ratio. For a thick panel, CLT $1 a$ generally shows lower values of deflection than $1 b$ which has opposite lamination of transverse layers. Configuration 2 leads to the highest values of deflection at low slenderness ratio, due to the considerable thickness of its central shear-compliant layers. When the bending effects become dominant, the configuration 2 shows lower values of deflection than the panels with five layers. This derives from the greater thickness of its upper and lower longitudinal layers (the middle one does not really contribute) than in CLT 5-ply. For the same reason the plate 3 with three layers presents generally the lowest val-

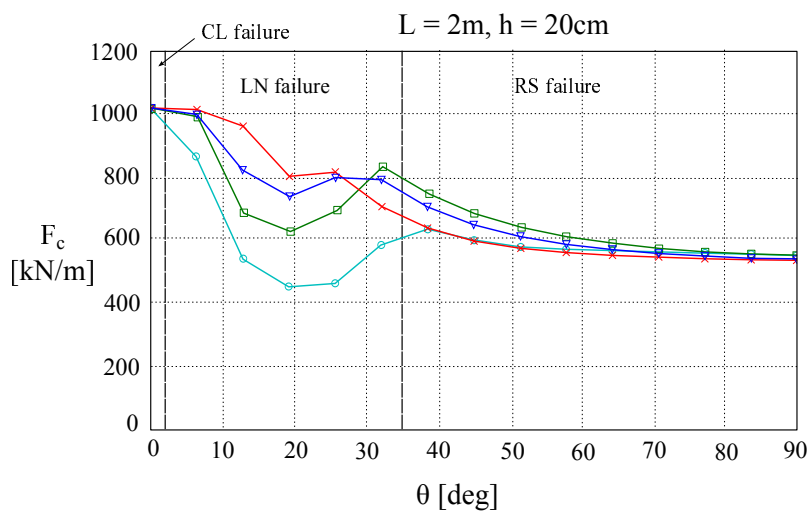

ues of deflection at every slenderness ratio when varying its transverse layer's orientation.

Figure 11 presents the variation of failure load $F_{c}$ and failure mode as a function of the orientation of transverse layers. While for a dominant shear regime, varying transverse layer orientation leads to an increasing failure load (Fig. 11-left), when dealing with a slender CLT, the effect of rotating cross layers becomes less significant (Fig. 11-right). Interestingly, and contrary to the deflection case, the difference between failure load trends for solutions $1 a$ and $1 b$ is significant, especially at a low slenderness ratio. This means that imposing an opposite lamination of transverse layers leads to higher shear strengths while changing their orientation. At values of $\theta$ between about $10^{\circ}$ and $40^{\circ}$, all the CLT configurations of both slenderness present sharp drops in the failure load. Further analysis proved that in this range of $\theta$, the transverse layers are submitted to torsional effects which produce high in-plane shear stresses, leading to an unexpected plane shear failure $(\mathrm{LN})$ of layers.

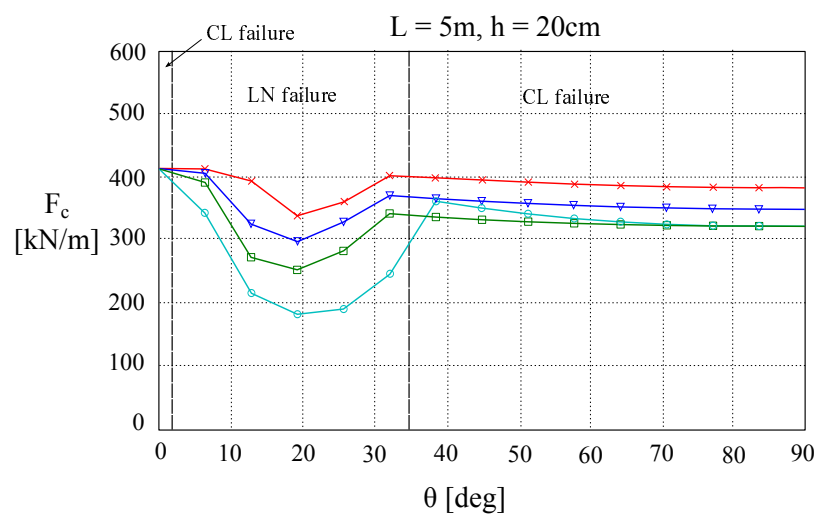

Fig. 11: Variation of failure load $F_{c}$ as a function of layer orientation for slenderness ratios of 10 (left) and 25 (right) 
Not surprisingly, considering the uni-axial bending configuration, the Glulam-like plate lay-up having all layers parallel to the bending direction, returns the best bending behavior. The favorable effect of rotating the transverse layers on the CLT mid-span deflection is more evident at low slenderness ratios, which are not very common in practical applications. Only thick CLT plates show increasing failure loads when rotating their transverse layers up to about $40^{\circ}$. After that lamination angle, plane shear stresses within cross layers increase drastically and lead to a failure load drop. Unless dealing with a thick panel and a dimensioning criterion driven by deflection, the low gains when varying transverse layer orientation make these configurations awkward to exploit.

\section{Conclusion and perspectives}

In the present paper, the bending behavior of CLT panels has been modeled by means of an equivalent layer model combined with a 3D elastic solution and a failure criterion. The heterogeneities at layer scale are taken into account by means of a homogeneous and equivalent layer whose mechanical properties change whether or not the narrow boards are glued to each other. Concerning the heterogeneities at the structure's scale, the exact 3D solution for layered plates in bending provides an accurate description of stresses, especially under concentrated loads. Moreover, such a solution leads to the analysis of non-orthotropic lay-ups and requires reasonable computational times. A failure criterion for wood combined with the elastic stress field has been used to identify progressive failure stages highlighted by the experimental evidence. The plate stiffnesses and failure modes predicted with the continuous and discontinuous equivalent layers are in agreement with the corresponding actual glued or unglued stages. Edge-glued layers make the panel a little stiffer but introduce also an additional failure mode at low load levels. Therefore it appears that gluing narrow boards has almost no positive effects on the mechanical response of CLT panels in bending. Moreover, clear wood mechanical properties lead to an accurate prediction, even if the tested reference panel was affected by the presence of knots. This shows that assembling lumber boards in a CLT configuration increases raw wood stiffness and strength, especially tensile one. The discontinuous equivalent-layer gives a good description of both elastic and failure response and therefore is used to study the influence of some panels' parameters on the CLT bending response. The trend of failure load and shear deflection as a function of the panel's slenderness ratio clearly quantify the influence of shear effects in CLT in bending. However, this shear weakness does not only depend on the panel's slenderness, but also on the CLT lay-up. Concerning the variation in the number of layers for a fixed total thickness, the more the number of layers increases, the more the mid-span deflection increases and the failure load decreases. This means that "homogenizing" CLT panels in uni-axial bending yields a worse mechanical behavior from a deterministic point of view. Finally, CLT shear weakness can be mitigated by varying the lamination of cross layers, especially in terms of deflection and dealing with thick plates. However, the small gains in terms of uni-axial bending performance make these further CLT configurations not really interesting.

Note that the discontinuous equivalent layer suggested in this paper involves simplified hypotheses on the layer's in-plane shear stiffness $\left(G_{L N}\right)$ and Young's modulus $\left(E_{N}\right)$. The actual reduction of in-plane stiffnesses of a non edgeglued CLT panel can be significant, as pointed out by theoretical (Moosbrugger et al 2006) and experimental (Brandner et al 2015) studies, but it is difficult to predict with simplified approaches. Therefore the hypotheses on the discontinuous layer's in-plane properties will be further investigated with a model currently in development (Lebée and Sab 2012; 2013). This model can also predict the influence of stronger heterogeneities like periodic voids within the panel and filled by insulating material, in order to obtain lighter and more acoustically efficient floors for mid-rise and highrise timber buildings.

Acknowledgements The authors gratefully acknowledge the research team composed of G. Hochreiner, J. Fussl and J. Eberhardsteiner (Vienna University of Technology, Institute for the mechanics of materials and structures) for sharing their experimental data.

\section{References}

Aicher S, Dill-Langer S (2000) Basic considerations to rolling shear modulus in wooden boards. Tech. rep., Otto Graf Journal

ANSI/APA (2012) ANSI/APA PRG 320-2012 Standards for performance-rated cross-laminated timber. ANSI/APA, Tacoma, US

Blass H, Fellmoser P (2004a) Design of solid wood panels with cross layers. In: Proocedings of the 8th World Conference on Timber Engineering

Blass H, Fellmoser P (2004b) Influence of rolling shear modulus on strength and stiffness of structural bonded timber elements. In: International Council for Research and Innovation in Building and Construction, Working Commission W18

Blass H, Gorlacher R (2000) Rolling shear in structural bonded timber elements. In: International conference on wood and wood fiber composites

Brandner R, Dietsch P, Droscher J, Shulte-Wrede M, Kreuzinger H, Sieder M, Schickhofer G, Winter S (2015) Shear properties of Cross Laminated Timber (CLT) under in-plane load: test configuration and experimental study. 
In: Proceedings of the 2nd International Network on Timber Engineering Research meeting

Cabrero JM, Blanco C, Gebremedhin KG, Martin-Meizoso A (2012) Assessment of phenomenological failure criteria for wood. European Journal of Wood and Wood Products 127(6):871-882

Chen Y, Lam F (2013) Bending performance of box-based Cross-Laminated Timber systems. Journal of Structural Engineering 139(12)

Czaderski C, Steiger R, Howald M, Olia S, Gulzow A, Niemz P (2007) Tests and calculations on 3-layered crosslaminated solid wood panels supported at all edges. European Journal of Wood and Wood Products 65:383-402

Dahl K (2009) Mechanical properties of clear wood from Norway spruce. PhD thesis, Norwegian University of science and technology

DIN (2004) DIN 1052:2004 Design rules for timber buildings (in German). German Institute of Standardization, Berlin, Germany

Eberhardsteiner J (2002) Mechanical behavior of Spruce (in German). Springer, Vienna, Austria

EN (2004) Eurocode 5 Design of timber structures. Part 1-1: General Common rules and rules for buildings. European Committee for Standardization, CEN, Bruxelles, Belgium

EN (2009) EN 338: Structural timber - strength classes. European Committee for Standardization, CEN, Bruxelles, Belgium

ETA (2013) ETA - 06/0009: Binderholz Cross Laminated Timber BBS. Binderholz Bausysteme GmbH, Zillertalstrasse 396263 Fugen, Austria

Gagnon S, Pirvu C (2013) CLT handbook: Cross Laminated Timber. FPInnovations, Qubec, Canada

Hochreiner G, Fussl J, Eberhardsteiner J (2013) Cross Laminated Timber plates subjected to concentrated loading. Experimental identification of failure mechanisms. Strain 50(1):68-71

Hochreiner G, Fussl J, Serrano E, Eberhardsteiner J (2014) Influence of wooden board strength class on the performance of Cross Laminated Timber plates investigated by means of full-field deformations measurements. Strain 50(2):161-173

Jobstl R, Moosbrugger T, Bogensperger T, Schickhofer G (2006) A contribution to the design and system effect of cross laminated timber (CLT). In: International Council for Research and Innovation in Building and Construction, Working Commission W18

Keunecke D, Hering S, Niemz P (2008) Three-dimensional elastic behaviour of common yew and Norway spruce. Wood Science and Technology 42(8):633-647

Kirchhoff G (1850) On the equilibrium and kinematics of an elastic plate (in German). Journal of Pure and Applied Mathematics 40:51-88
Kreuzinger H (1999) Plate and shell structures. A model for common calculation tools (in German). Bauen mit Holz 1:34-39

Lebée A, Sab K (2011) A Bending-Gradient model for thick plates, Part I: Theory. International Journal of Solids and Structures 48:2878-2888

Lebée A, Sab K (2012) Homogenization of thick periodic plates: Application of the Bending-Gradient plate theory to a folded core sandwich panel. International Journal of Solids and Structures 49:2778-2792

Lebée A, Sab K (2013) Homogenization of a space frame as a thick plate: Application of the Bending-Gradient theory to a beam lattice. Computer and Structures 70:88-101

March HW (1936) Bending of a Centrally Loaded Rectangular Strip of Plywood. Physics (College Park Md) 7(1), DOI 10.1063/1.1745342

Mestek P (2011) Cross laminated timber panels under concentrated loads: design with local shear reinforcement (in german). PhD thesis, Munich University of Technology

Mestek P, Kreuzinger H, Winter S (2008) Design of Cross Laminated Timber (CLT). In: Proceedings of the 10th World Conference on Timber Engineering

Moosbrugger T, Guggenberger W, Bogensperger T (2006) Cross Laminated Timber wall segments under homogeneous shear with and without openings. In: Proceedings of the 9th World Conference on Timber Engineering

Pagano N (1969) Exact solutions for rectangular bidirectional composites and sandwich plates. Journal of composite materials 4:20-34

Pagano N (1970) Influence of shear coupling in cylindrical bending of anisotropic laminates. Journal of composite materials 4:330-343

van der Put T (1982) A general failure criterion for wood. In: Proceedings of 15th Conseil Industriel des BoisInternational. Union of Forestry Research Organizations Meeting, Boras, Sweden

Schickhofer G, Bogensperger T, Moosbrugger T (2009) CLT Handbook: buildings with Cross Laminated Timber (in German). Technische Universitat Graz, Graz, Austria

Sebera V, Muszyski L, Tippner J, Noyel M, Pisaneschi T, Sundberg B (2013) FE analysis of CLT panel subjected to torsion and verified by DIC. Materials and Structures 1(9)

Stapel P, van de Kuilen J (2014) Influence of cross-section and knot assessment on the strength of visually graded Norway spruce. European Journal of Wood and Wooden Products 72:213-227

Sturzenbecher R, Hofstetter K, Eberhardsteiner J (2010) Structural design of Cross Laminated Timber (CLT) by advanced plate theories. Composite science and technology 70:1368-1379

Thai ND, DOttavio M, Caron JF (2013) Bending analysis of laminated and sandwich plates using a layer-wise stress model. Composite Structures 96:135-142 
Tsai S, Wu E (1971) A general theory of strength for anisotropic materials. Journal of composite materials 5(1):58-80

Zhou Q, Gong M, Chui Y, Mohammad M (2014) Measurement of rolling shear modulus and strength of Cross Laminated Timber using bending and two plates shear tests. Wood and fiber science 46(2):1-11 